\title{
Numerical Evaluation of a Decentralised Feedforward Active Control System for Electrical Transformer Noise
}

\author{
Júlio A. Cordioli \\ Federal University of Santa Catarina, Rua Gustavo Barroso, 271 Estreito, Florianópolis, SC CEP 88070-490, Brazil
}

\author{
Colin H. Hansen ${ }^{\dagger}$, Xun Li and Xiaojun Qiu ${ }^{\dagger}$ \\ Department of Mechanical Engineering, Adelaide University, S.A. 5005, Australia
}

(Received 30 May 2001; accepted 28 September 2001)

\begin{abstract}
The implementation of a decentralised architecture for an active system to control electrical transformer noise is discussed and a general theoretical analysis of the performance and convergence of a decentralised system is developed. Using the equations derived in the analysis, together with transfer function and noise level data measured for a large electrical transformer, the performance and the convergence characteristics are calculated and compared for different control system configurations. It is demonstrated that the behaviour of a decentralised system with a leakage coefficient incorporated can be very different from the behaviour of a fully coupled system.
\end{abstract}

${ }^{\dagger}$ Member of the International Institute of Acoustics and Vibration (IIAV)

\section{INTRODUCTION}

An active noise control system for reducing the periodic noise radiated by a large electrical transformer usually consists of a large number of control sources and error sensors distributed over a large area. This results in two problems in the design of the system: the electrical connection between sensors, the control sources and the controller requires a large amount of wiring; and the control algorithm requires considerable processing power, both of which increase the cost of the final system. In addition, the convergence speed of an active control system decreases as the number of channels increases and the $\mathrm{CPU}$ processing requirements increase exponentially. ${ }^{\mathbf{1} 2}$

One way to minimise these problems is to partition the system into a number of independent controllers, where each individual controller is responsible for driving a small number of control sources using a small number of error sensors. Such a system is known as a decentralised or decoupled system. Previous theoretical work ${ }^{4}$ has shown that it is possible to achieve reasonable results using a decentralised system. The previous theoretical study demonstrated that a fully decentralised system using the same number of control sources and error sensors, where each control source is adjusted using the response of only one error sensor, would perform at a level similar to that of a centralised system. However on-site measurements show that this result is not necessarily true in practice and that decoupling the system can considerably degrade its performance.

This paper is concerned with investigating the viability of decentralising an active noise control system for a large electrical transformer. A general analysis of a feedforward control system at a single frequency is presented, in which the equations for the steady-state performance and the convergence characteristics of the centralised and decentralised sys- tem are derived. The data from a large electrical transformer are used to calculate the performance of the centralised system and to evaluate different possibilities for partitioning the system.

\section{THEORETICAL STUDY}

\subsection{Multiple Input and Multiple Output Controller - Steady-state Performance}

A generalisation of an active system for the control of electrical transformer noise is illustrated in Fig. 1.

The system includes an adaptive feedforward controller where a set of error sensors is used to adjust the complex strengths of a set of control sources. Inertial actuators are used as control sources to modify the vibration response of the transformer tank and hence attenuate the radiated sound. For real time adaptive control, the reference signal was obtained from a coil wrapped around the primary power cable. This a provided synchronised $50 \mathrm{~Hz}$ signal that was converted, using conditioning electronics, to the required 100 , 200, 300 and $400 \mathrm{~Hz}$ harmonics that are responsible for the majority of the radiated sound. However, for the simulations undertaken here in the frequency domain, a reference signal is not needed as it implicit in the formulation.

The analysis outlined here is carried out in the frequency domain and the excitation is assumed to be tonal. For a single centralised multi-channel system using all error sensors and control actuators, the $\left(N_{e} \times 1\right)$ vector of error signals can be expressed as:

$$
e=p+Z f
$$

where $\boldsymbol{p}$ is the vector of the primary sound pressures as measured by the error microphones, $\boldsymbol{Z}$ is the $\left(N_{e} \times N_{c}\right)$ matrix of the transfer functions between the actuators and the error microphones and $\boldsymbol{f}$ is the $\left(N_{c} \times 1\right)$ vector of the inputs to the actuators. 